\title{
Tripartite motif-containing 11 regulates the proliferation and apoptosis of breast cancer cells
}

\author{
XIANPING DAI ${ }^{*}$, FENG GENG ${ }^{*}$, MENGSHUN LI and MING LIU \\ School of Pharmacy, Binzhou Medical University, Yantai, Shandong 264003, P.R. China
}

Received October 11, 2017; Accepted September 28, 2018

DOI: 10.3892/or.2019.7015

\begin{abstract}
Breast cancer, an increasing health problem worldwide, is the second major cause of cancer-associated mortality in females. Studies have focused on the pathogenesis of breast cancer for decades, but the underlying mechanisms have not been fully elucidated. Tripartite motif-containing 11 (TRIM11), a novel oncogene that was recently identified, was reported to function in various types of cancer, including ovarian and lung cancer. In the present study, high expression levels of TRIM11 were detected in breast cancer tissues by reverse transcription-quantitative polymerase chain reaction and western blot analysis, which suggested that TRIM11 was likely to function in the progression of breast cancer. Downregulation of TRIM11 in MCF-7 and MDA-MB-231 cells inhibited cell proliferation and promoted cell apoptosis, accompanied by increased phosphatase and tensin homolog deleted on chromosome 10 (PTEN), p53 and Bcl-2-associated $\mathrm{X}$ protein, and decreased B-cell lymphoma 2, phosphorylated c-Jun N-terminal kinase 1/2 (p-JNK1/2) and phosphorylated extracellular signal-regulated kinases $1 / 2$ (p-ERK1/2), whereas the overexpression of TRIM11 completely reversed these effects. Furthermore, TRIM11 downregulation enhanced the pro-apoptotic effect of chemotherapy drugs on breast cancer cells, and high levels of TRIM11 expression were observed in cisplatin- and paclitaxel-resistant breast cancer tissues. These data indicated that TRIM11 is crucial to the development of breast cancer, and TRIM11 downregulation may benefit the treatment of breast cancer by regulating ERK1/2 and JNK1/2 signaling and the expression of apoptosis-associated genes.
\end{abstract}

Correspondence to: Mr. Xianping Dai, School of Pharmacy, Binzhou Medical University, 346 Guanhai Road, Yantai, Shandong 264003, P.R. China

E-mail: daixianping100@163.com

${ }^{*}$ Contributed equally

Key words: tripartite motif-containing 11, breast cancer, proliferation, apoptosis, chemotherapy drugs

\section{Introduction}

Breast cancer is a malignant tumor that occurs in the mammary glandular epithelium. Similar to ovarian cancer, breast cancer also causes great harm to the health of females, with $99 \%$ of breast cancer diagnoses occurring in females and only $1 \%$ of diagnoses occurring in males (1). Therefore, breast cancer is regarded as a common cause of cancer-associated mortality in females. The global incidence of breast cancer has been increasing since the late 1970s. The majority of the patients who are diagnosed with early-stage breast cancer can be cured by surgery, but surgery is unable to completely prevent the metastasis of tumors $(2,3)$.

Over recent decades, a variety of drugs have been developed for the treatment of breast cancer. Cisplatin, first developed in 1845 (4), is regarded as the first-line chemotherapy option, not only in breast cancer, but also in other types of cancer, including ovarian cancer (5). Paclitaxel, another drug isolated in 1962 (6-8), has similar effects to cisplatin on cancer types, including breast and ovarian cancer (9). In addition, adriamycin (doxorubicin) $(10,11)$, gemcitabine (12) and fluorouracil (5-FU) (13) are also used as chemotherapeutic agents for the clinical treatment of breast and lung cancer, and other types of cancer. Nonetheless, due to the development of drug resistance, chemotherapy cannot always cure breast cancer or other types of cancer, and is likely to be accompanied by the recurrence of cancer metastasis (14).

Tripartite motif-containing 11 (TRIM11) is a member of the TRIM family, which was identified to contain E3 ubiquitin ligases. TRIM proteins were characterized by the presence of a tripartite motif consisting of three conserved domains, including RING, B-box and a coiled-coil region $(15,16)$. Recent studies have demonstrated that TRIM11 is an oncogene and is involved in several types of cancer, including lung cancer (17), colon cancer (18) and ovarian cancer (19). For example, the downregulation of TRIM11 in ovarian cancer inhibited the proliferation and invasion of tumor cells (19), and increasing TRIM11 expression in lung cancer was correlated with a poor prognosis of patients (17). Additionally, studies have associated the abnormal expression of TRIM proteins with the development of breast cancer (20-22). For example, the expression of TRIM24 was significantly increased in breast cancer tissues, and its overexpression was associated with a poor prognosis of patients with breast cancer $(20,21)$. Furthermore, it was reported that TRIM29 functioned as a tumor suppressor 
in breast cancer (22). However, little is known regarding the changes in the expression and function of TRIM11 in breast cancer.

In the present study, elevated TRIM11 levels were detected in breast cancer tissues, which suggested that TRIM11 may be involved in breast cancer progression. In MCF-7 and MDA-MB-231 cells, TRIM11 downregulation suppressed cell proliferation and accelerated apoptosis, whereas upregulation of TRIM11 had the opposite effect, which was accompanied by significant alterations in apoptosis-associated genes. Furthermore, TRIM11 downregulation enhanced the pro-apoptotic effect of chemotherapy drugs on breast cancer cells, and high TRIM11 expression was observed in cisplatin- and paclitaxel-resistant breast cancer tissues. Therefore, it was hypothesized that the downregulation of TRIM11 may be beneficial for the treatment of breast cancer.

\section{Materials and methods}

Breast cancer tissues and adjacent normal tissues. A total of 30 patients with breast cancer treated at the Affiliated Hospital of Binzhou Medical University (Yantai, China) were enrolled in the present study. The mean age of the patients was 55 years (age range, 50-65 years). Following the receipt of written informed consent, paired tumor and adjacent normal tissues were collected from the patients between June 6 and December 15, 2016 and immediately frozen and stored in liquid nitrogen at $-196^{\circ} \mathrm{C}$ prior to use. Following the extraction of total RNA and proteins from these samples, the expression of TRIM11 was detected by reverse transcription-quantitative polymerase chain reaction (RT-qPCR) and western blot analysis. All experiments conducted in the present study were approved by the Ethics Committee of Binzhou Medical University.

Cell culture. Human breast cancer MCF-7 and MDA-MB-231 cell lines were obtained from Type Culture Collection of the Chinese Academy of Science (Shanghai, China) and cultured in Dulbecco's modified Eagle's medium (DMEM; HyClone; GE Healthcare Life Sciences, Logan, UT, USA) containing 10\% fetal bovine serum (Gibco; Thermo Fisher Scientific, Inc., Waltham, MA, USA) and 1\% antibiotic (a mixture of penicillin and streptomycin; Beijing Solarbio Science \& Technology Co., Ltd., Beijing, China) in a $5 \% \mathrm{CO}_{2}$ incubator (Thermo Fisher Scientific, Inc., Waltham, MA, USA) at $37^{\circ} \mathrm{C}$. During the period of incubation, the medium was changed every two days.

Construction of the lentivirus. TRIM11 short hairpin RNA (shRNA) (3'-CAGAAGTTGTGCCTATGGA-5') and a negative control shRNA (3'-CCTAAGGTTAAGTCGCCCTCG-5') were applied to construct RNA interference lentiviruses. The coding DNA sequence region of TRIM11 synthesized by Genewiz, Inc. (South Plainfield, NJ, USA) was inserted into EcoRI/BamHI restriction sites of a pLVX-AcGFP1-C1 plasmid and confirmed by DNA sequencing (Shanghai Majorbio Pharmaceutical Technology Co., Ltd., Shanghai, China). Lentiviral constructs of pLKO.1-shTRIM11 (1,000 ng), pLVX-AcGFP1-C1-TRIM11 (1,000 ng) or pLVX-AcGFP1-C1, and pLKO.1 (Addgene, Inc., Cambridge, MA, USA) empty vectors were co-transfected with psPAX2 and pMD2.G (Addgene, Inc.) viral packaging plasmids into 293T cells by Lipofectamine 2000 (Invitrogen;
Thermo Fisher Scientific, Inc.). Following transfection for $48 \mathrm{~h}$, the viral supernatant that was used to infect MCF-7 and MDA-MB-231 cells was collected (17).

Division of the experimental groups. To regulate TRIM11 expression in the MCF-7 and MDA-MB-231 cells, lentivirus-mediated RNA interference and overexpression were used, and four groups of cultured cells were determined: The first group consisted of wild-type cells cultured with DMEM (Control); the second group consisted of cells infected with the negative control (NC) lentivirus; the third group consisted of cells infected with the TRIM11 shRNA lentivirus (shTRIM11); and the fourth group consisted of cells infected with the TRIM11 recombinant lentivirus (oeTRIM11). Following infection for $0,24,48$ or $72 \mathrm{~h}$, proliferation assays were performed, and RT-qPCR and western blot analyses were performed, as well as apoptosis assays $48 \mathrm{~h}$ later.

To further investigate the impact of TRIM11 on breast cancer, following downregulation of TRIM11, the cells were treated with $5 \mu \mathrm{g} / \mathrm{ml}$ cisplatin, paclitaxel, adriamycin, 5-FU or gemcitabine individually for $24 \mathrm{~h}$. In addition, the apoptosis rates of MCF-7 and MDA-MB-231 cells were detected using flow cytometric analysis.

Proliferation assay. Cells in the logarithmic growth phase were digested by $0.25 \%$ trypsin (Beijing Solarbio Science \& Technology Co., Ltd.), inoculated in 96-well culture plates at a density of $3 \times 10^{3}$ cells/well and then cultured at $37^{\circ} \mathrm{C}$ in a $5 \% \mathrm{CO}_{2}$ humidified incubator overnight. On the next day, according to the aforementioned experimental grouping, the cells were infected with lentivirus for $0,24,48$ and $72 \mathrm{~h}$. Subsequently, $100 \mu 1$ 10\% Cell Counting Kit-8 (CCK-8; SAB Biotherapeutics, Inc., Sioux Falls, SD, USA) solution in serum-free DMEM was added to each well and incubated for $1 \mathrm{~h}$. Finally, the optical density of the absorbance at $450 \mathrm{~nm}$ was measured using a microplate reader (Perlong Medical Equipment Co., Ltd., Beijing, China).

$R N A$ extraction and $R T-q P C R$. Following extraction from tissue samples or lentivirus-infected MCF-7 cells by TRIzol (Invitrogen; Thermo Fisher Scientific, Inc.), total RNA was quantified and then $1 \%$ agarose gel electrophoresis was used to confirm the integrity of the isolated RNA. Subsequently, isolated RNA was reverse transcribed into cDNA with a procedure of $37^{\circ} \mathrm{C}$ for $30 \mathrm{~min}, 85^{\circ} \mathrm{C}$ for $5 \mathrm{~min}$ and $4^{\circ} \mathrm{C}$ for 5 min using a reverse transcriptase kit (Fermentas; Thermo Fisher Scientific, Inc.). RT-qPCR reactions were conducted on an ABI Prism 7300 instrument (Applied Biosystems; Thermo Fisher Scientific, Inc.) with a SYBR-Green PCR kit (Thermo Fisher Scientific, Inc.). Subsequently, the mRNA level of TRIM11 normalized to GAPDH was analyzed by the $2^{-\Delta \Delta \mathrm{Cq}}$ method (23). The primer sequences used were as follows: TRIM11 forward, 5'-GAGAACGTGAACAGGAAG GAG-3' and reverse, 5'-CCATCGGTGGCACTGTAGAA-3'; GAPDH forward, 5'-CACCCACTCCTCCACCTTTG-3' and reverse, 5'-CCACCACCCTGTTGCTGTAG-3'. In addition, the thermocycling conditions were as follows: $95^{\circ} \mathrm{C}$ for $10 \mathrm{~min}$, followed by 40 cycles of $95^{\circ} \mathrm{C}$ for $15 \mathrm{sec}$ and $60^{\circ} \mathrm{C}$ for $45 \mathrm{sec}$; $95^{\circ} \mathrm{C}$ for $15 \mathrm{sec}$ and $60^{\circ} \mathrm{C}$ for $1 \mathrm{~min}$ for one cycle; and $95^{\circ} \mathrm{C}$ for $15 \mathrm{sec}$ and $60^{\circ} \mathrm{C}$ for $15 \mathrm{sec}$ for one cycle (24). 
A

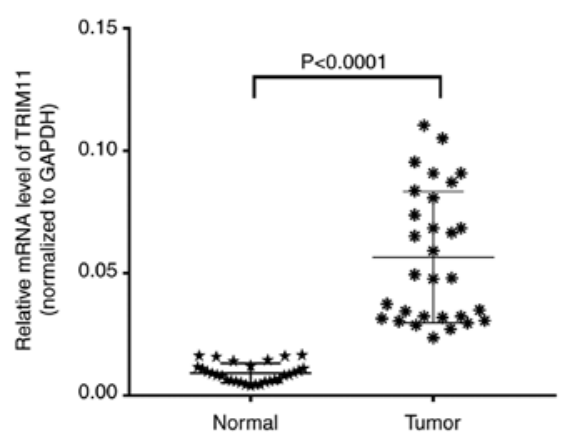

B
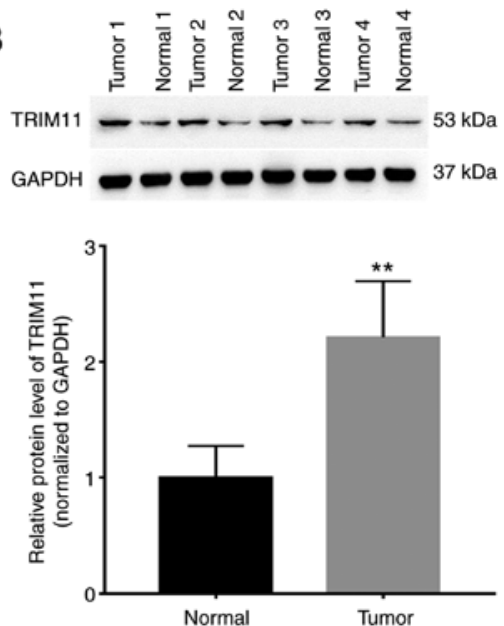

Figure 1. TRIM11 expression is significantly increased in the tumor tissues of patients with breast cancer. Tumor and adjacent normal tissues were obtained from 30 patients with breast cancer. (A) The mRNA expression level of TRIM11 in the tumor and adjacent normal tissue samples was quantified by reverse transcription-quantitative polymerase chain reaction. (B) Western blot analysis was performed to quantify the protein expression level of TRIM11. Data are presented as the mean \pm standard deviation. ${ }^{* *} \mathrm{P}<0.01$, compared with normal tissues. TRIM11, tripartite motif-containing 11 .

Extraction of protein and western blot analysis. Radioimmunoprecipitation assay lysis buffer (Beijing Solarbio Science \& Technology Co., Ltd.) containing protease and phosphatase inhibitors was used to homogenize the cells or tissue samples by incubation at $4^{\circ} \mathrm{C}$ for $30 \mathrm{~min}$. Next, cells or tissue lysates were centrifuged at $12,000 \mathrm{xg}$ at $4^{\circ} \mathrm{C}$ for $10 \mathrm{~min}$, and the proteins in the supernatant were acquired and later quantified by a bicinchoninic acid protein quantification kit (Thermo Fisher Scientific, Inc.). SDS-PAGE (10\%; JRDUN Biotechnology Co., Ltd., Shanghai, China) was applied to separate the proteins $(25 \mu \mathrm{g})$, followed by semi-dry transfer onto polyvinylidene fluoride membranes (EMD Millipore, Billerica, MA, USA) by electroblotting. Subsequently, 5\% skimmed milk (BD Biosciences, Franklin Lakes, NJ, USA) was used to block the membranes for $1 \mathrm{~h}$ at room temperature. Next, the membranes were incubated with primary antibodies against TRIM11 (1:2,000; cat. no. Ab111694; Abcam, Cambridge, UK), tumor protein p53 [p53; 1:1,000; cat. no. 2524, Cell Signaling Technology (CST), Inc., Danvers, MA, USA], phosphatase and tensin homolog (PTEN; 1:1,000; cat. no. 9552; CST, Inc.), B-cell lymphoma 2 (Bcl-2; 1:200; cat. no. Sc-492; Santa Cruz Biotechnology, Inc., Dallas, TX, USA), Bcl-2-associated X protein (Bax; 1:300; cat. no. Sc-493; Santa Cruz Biotechnology, Inc.), phosphorylated c-Jun N-terminal kinase 1/2 (p-JNK1/2; 1:2,000; cat. no. 9255; CST, Inc.), JNK1/2 (1:1,000; cat. no. 9252; CST, Inc.), phosphorylated extracellular signal-regulated kinase 1/2 (p-ERK1/2; 1:1,000; cat. no. 9101; CST, Inc.), ERK1/2 (1:1,000; cat. no. 9102; CST, Inc.) or GAPDH (1:2,000; cat. no. 5174; CST, Inc.) at $4^{\circ} \mathrm{C}$ overnight with gentle agitating. Following 5-6 washes, the membranes were incubated with horseradish peroxidase (HRP)-labeled secondary antibodies (1:1,000; Beyotime Institute of Biotechnology, Haimen, China) of goat anti-rabbit (cat. no. A0208) and goat anti-mouse (cat. no. A0216) at $37^{\circ} \mathrm{C}$ for $1 \mathrm{~h}$. Finally, the membranes were incubated in the dark with immobilon western chemiluminescent horseradish peroxidase substrate (EMD Millipore) for $5 \mathrm{~min}$ and the protein bands were visualized under an enhanced chemiluminescence imaging system (Tanon-5200; Tanon Science and Technology
Co., Ltd., Shanghai, China). GAPDH as an endogenous reference gene, the relative protein expression levels were calculated by ImageJ software $1.47 \mathrm{v}$ (National Institutes of Health, Bethesda, MD, USA).

Cell apoptosis analysis. A flow cytometer (BD Biosciences) was used to detect the proportion of apoptotic cells. Treated cells were digested, resuspended and counted. Resuspended cells $(5,000,000-10,000,000)$ were centrifuged at $1,000 \mathrm{x} \mathrm{g}$ for $5 \mathrm{~min}$ to obtain the cell precipitants. Subsequently, the cells were resuspended gently with $195 \mu$ l binding buffer for Annexin V-fluorescein isothiocyanate (FITC) staining. Next, following incubation with $5 \mu \mathrm{l}$ Annexin V-FITC (Beyotime Institute of Biotechnology) for $15 \mathrm{~min}$ in the dark at $4^{\circ} \mathrm{C}, 5 \mu \mathrm{l}$ propidium iodide (PI) was added and cells were incubated for a further $5 \mathrm{~min}$ at $4^{\circ} \mathrm{C}$. Simultaneously, a tube without Annexin V-FITC or PI was used as a negative control. Subsequently, the cell apoptosis rates were determined by flow cytometry (FCM) and analyzed using BD Accuri ${ }^{\text {TM }}$ C6 Software (Version 1.0.264.21; BD Biosciences).

Statistical analysis. All the statistical analyses were performed using GraphPad Prism 7.0 software (GraphPad Software, Inc., La Jolla, CA, USA). Student's t-tests were performed to assess the quantitative data compared between two groups, and one-way analysis of variance was used for multiple comparisons. All data were based on at least three independent experiments and values are presented as the mean \pm standard deviation. $\mathrm{P}<0.05$ was considered to indicate a statistically significant difference.

\section{Results}

TRIM11 expression is significantly increased in the tumor tissues of patients with breast cancer. A total of 30 paired tumor and normal tissues of patients with breast cancer were collected, and RT-qPCR and western blot analysis were applied to quantify TRIM11 expression levels in these tissues. As demonstrated in Fig. 1, statistical analysis by Student's t-tests 
A

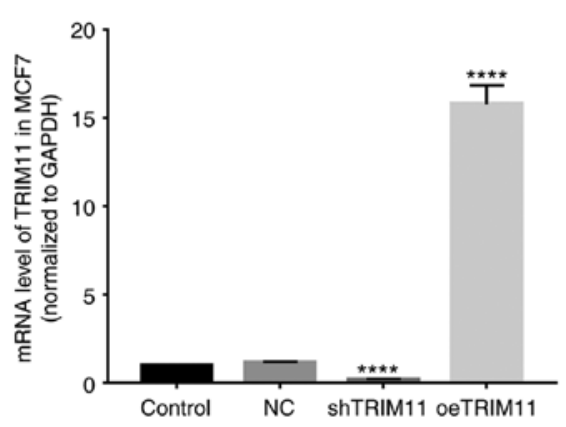

C

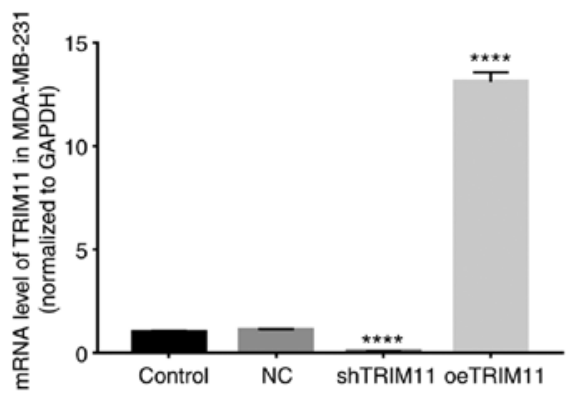

B
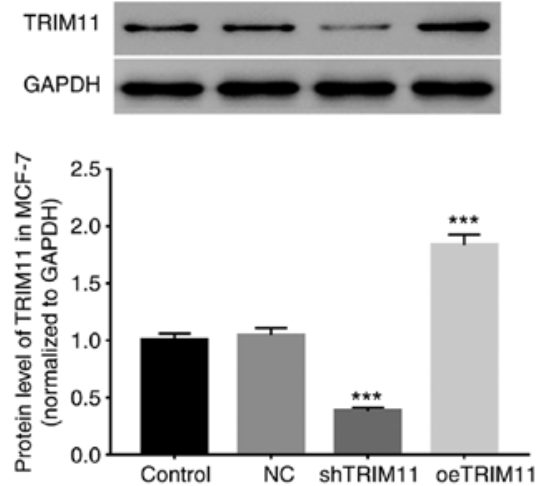

D TRIM11

GAPDH

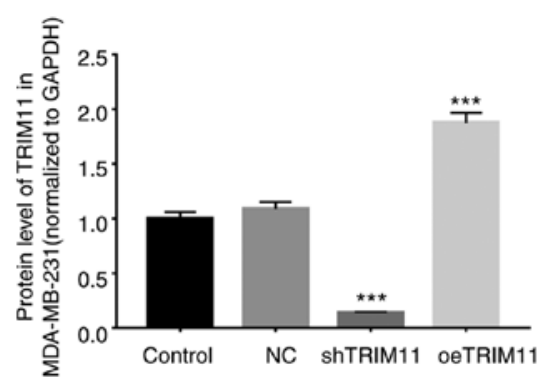

Figure 2. Downregulation and upregulation of TRIM11 in MCF-7 and MDA-MB-231 cell lines. Packaged lentiviruses were used to infect MCF-7 and MDA-MB-231 cells, and then cells were harvested after $48 \mathrm{~h}$ of infection. The TRIM11 (A) mRNA and (B) protein expression levels in MCF-7 cells and the TRIM11 (C) mRNA and (D) protein expression levels in MDA-MB-231 cells were detected. Data are presented as the mean \pm standard deviation. ${ }^{* * *} \mathrm{P}<0.001$,

${ }^{* * * * *} \mathrm{P}<0.0001$, compared with the NC. TRIM11, tripartite motif-containing 11; NC, negative control; sh, short hairpin RNA; oe, overexpression.

\section{A}

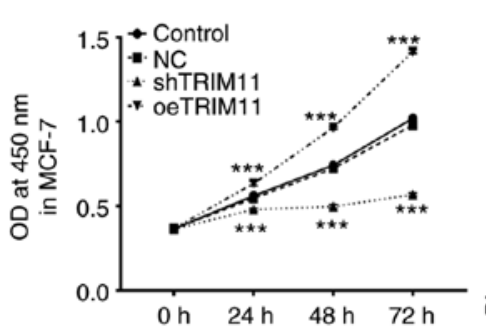

C

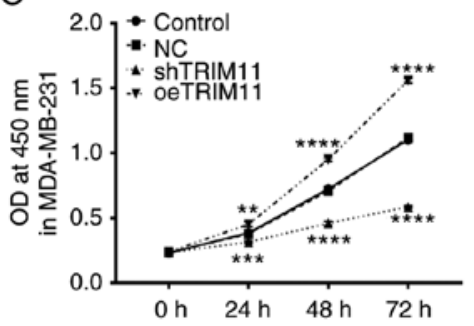

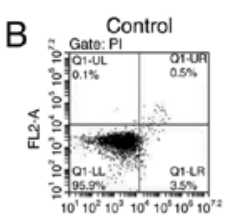
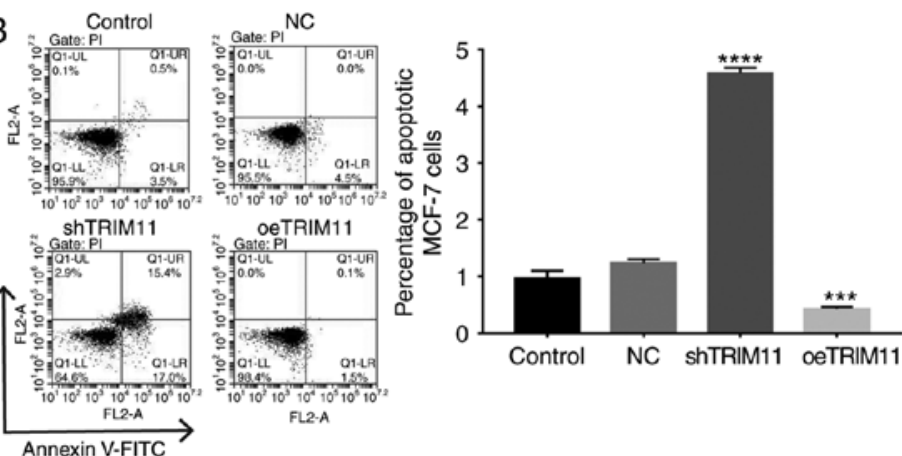

D

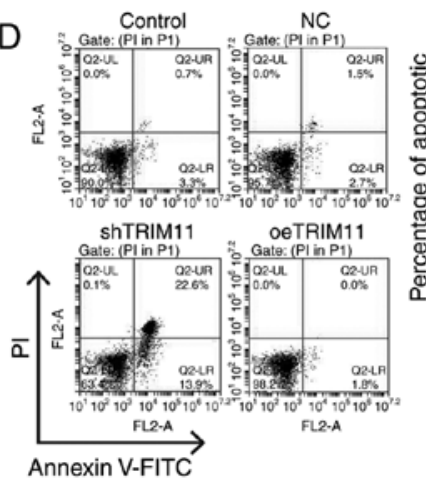

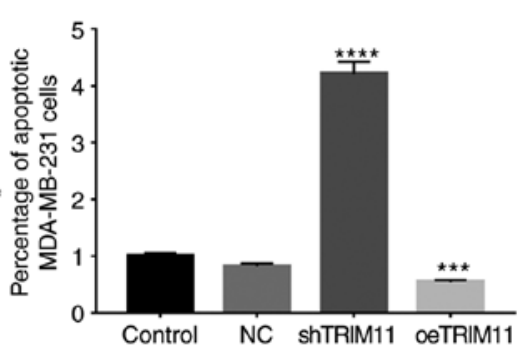

Figure 3. The effects of TRIM11 on the viability and apoptosis of MCF-7 and MDA-MB-231 cells. After 0, 24, 48 and $72 \mathrm{~h}$ of lentiviral infection, cell proliferation rates of (A) MCF-7 and (C) MDA-MB-231 cells were evaluated by Cell Counting Kit-8 assays. After 48 h, apoptotic cells in (B) MCF-7 and (D) MDA-MB-231 cells were detected by flow cytometric analysis using Annexin V-FITC/PI double staining. Early apoptotic cells that were stained with Annexin-V but not with PI are in the lower right quadrant, late apoptotic cells or necrotic cells that were stained the two dyes are in the upper right quadrant, while the lower left quadrant represents live cells. All data are presented as the mean \pm standard deviation. ${ }^{* * *} \mathrm{P}<0.01,{ }^{* * * *} \mathrm{P}<0.001,{ }^{* * * * *} \mathrm{P}<0.0001$, compared with the NC. OD, optical density; TRIM11, tripartite motif-containing 11; FITC, fluorescein isothiocyanate; PI, propidium iodide; NC, negative control; sh, short hairpin RNA; oe, overexpression. 

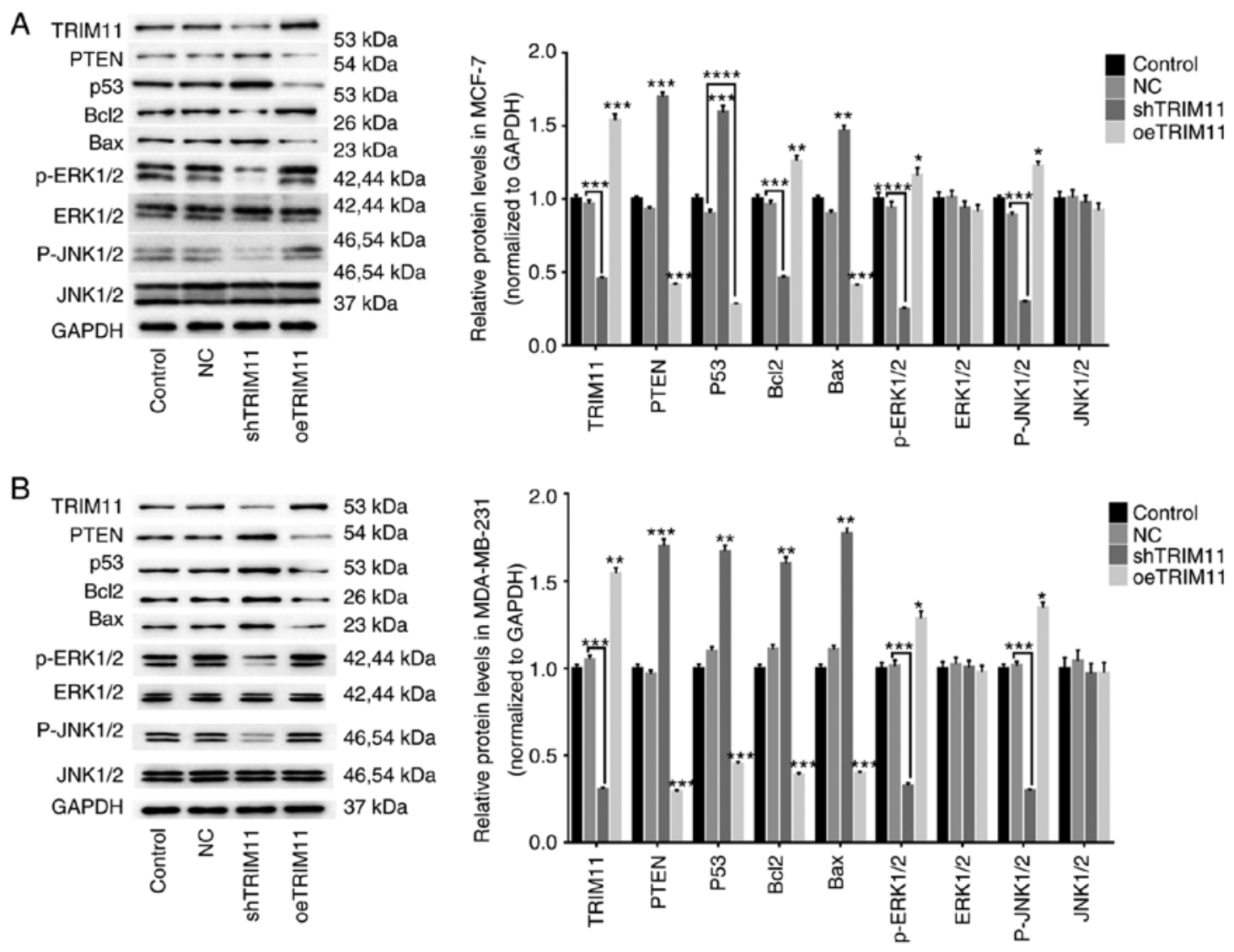

Figure 4. The expression changes of several associated genes following TRIM11 downregulation and upregulation. At $48 \mathrm{~h}$ after lentiviral infection, protein levels of TRIM11, PTEN, p53, Bcl2, Bax, p-ERK1/2, ERK1/2, p-JNK1/2, JNK1/2 in MCF-7 (A) and MDA-MB-231 (B) cells were quantified by western blot analysis. Data are expressed as the mean \pm standard deviation. ${ }^{*} \mathrm{P}<0.05,{ }^{* *} \mathrm{P}<0.01,{ }^{* * *} \mathrm{P}<0.001,{ }^{* * * * *} \mathrm{P}<0.0001$, compared with the NC. TRIM11, tripartite motif-containing 11 ; NC, negative control; sh, short hairpin RNA; oe, overexpression; PTEN, phosphatase and tensin homolog; p53, tumor protein p53; Bcl-2, B-cell lymphoma 2; Bax, Bcl-2-associated X protein; p-ERK1/2, phosphorylated extracellular signal-regulated kinase; p-JNK1/2, phosphorylated c-Jun N-terminal kinase 1/2.

revealed that mRNA (Fig. 1A) and protein (Fig. 1B) levels of TRIM11 were significantly higher in the breast cancer tissues than in the normal tissues, which indicated that TRIM11 may serve a pivotal role in the progression of breast cancer.

Downregulation and upregulation of TRIMII in MCF-7 and MDA-MB-231 cell lines. For further study, breast cancer MCF-7 and MDA-MB-231 cells were infected with TRIM11 interference and overexpression lentiviruses to downregulate and upregulate TRIM11 expression, respectively. After $48 \mathrm{~h}$ of infection, mRNA and protein expression levels of TRIM11 were quantified by RT-qPCR and western blot analysis, respectively. As demonstrated in Fig. 2, in MCF-7 (Fig. 2A and B) and MDA-MB-231 (Fig. 2C and D) cells, TRIM11 expression was significantly reduced when infected with the shTRIM11 lentivirus, whereas significantly increased expression was observed following infection with the oeTRIM11 lentivirus. Therefore, the TRIM11 lentiviruses were applied in the subsequent experiments due to their effective regulation of TRIM11 expression.

The effects of TRIM11 on the viability and apoptosis of MCF-7 and $M D A-M B-231$ cells. Following downregulation and upregulation of TRIM11 expression in MCF-7 and MDA-MB-231 cells, cell viability was evaluated by CCK- 8 assay, and FCM with Annexin V-FITC/PI staining was performed to detect cell apoptosis rates. As demonstrated in Fig. 3, TRIM11 silencing significantly decreased the cell proliferation rates of MCF-7 cells, whereas there was a significant increase in cell proliferation when TRIM11 was overexpressed (Fig. 3A). In addition, the effect of TRIM11 on MCF-7 cell apoptosis was opposite to its effect on the cell proliferation (Fig. 3B). Additionally, downregulation and upregulation of TRIM11 also had the same effect on the proliferation and apoptosis of MDA-MB-231 cells (Fig. 3C and D). These data demonstrated that TRIM11 has an effect on the growth and survival of breast cancer cells by promoting cell proliferation and inhibiting cell apoptosis.

The expression changes of several associated genes following TRIM11 downregulation and upregulation. The present study also further investigated the mechanism underlying the anti-apoptotic effect of TRIM11 downregulation on breast cancer cells. Following TRIM11 downregulation and upregulation, the protein levels of several associated genes were examined by western blot analysis. As demonstrated in Fig. 4, in MCF-7 (Fig. 4A) and MDA-MB-231 (Fig. 4B) cells, the expression of apoptosis-associated genes PTEN, p53 and Bax was significantly increased when TRIM11 was downregulated. By contrast, PTEN, p53 and Bax expression levels were markedly reduced following TRIM11 upregulation. In addition, the levels of Bcl-2, p-ERK1/2 and p-JNK1/2 were positively 

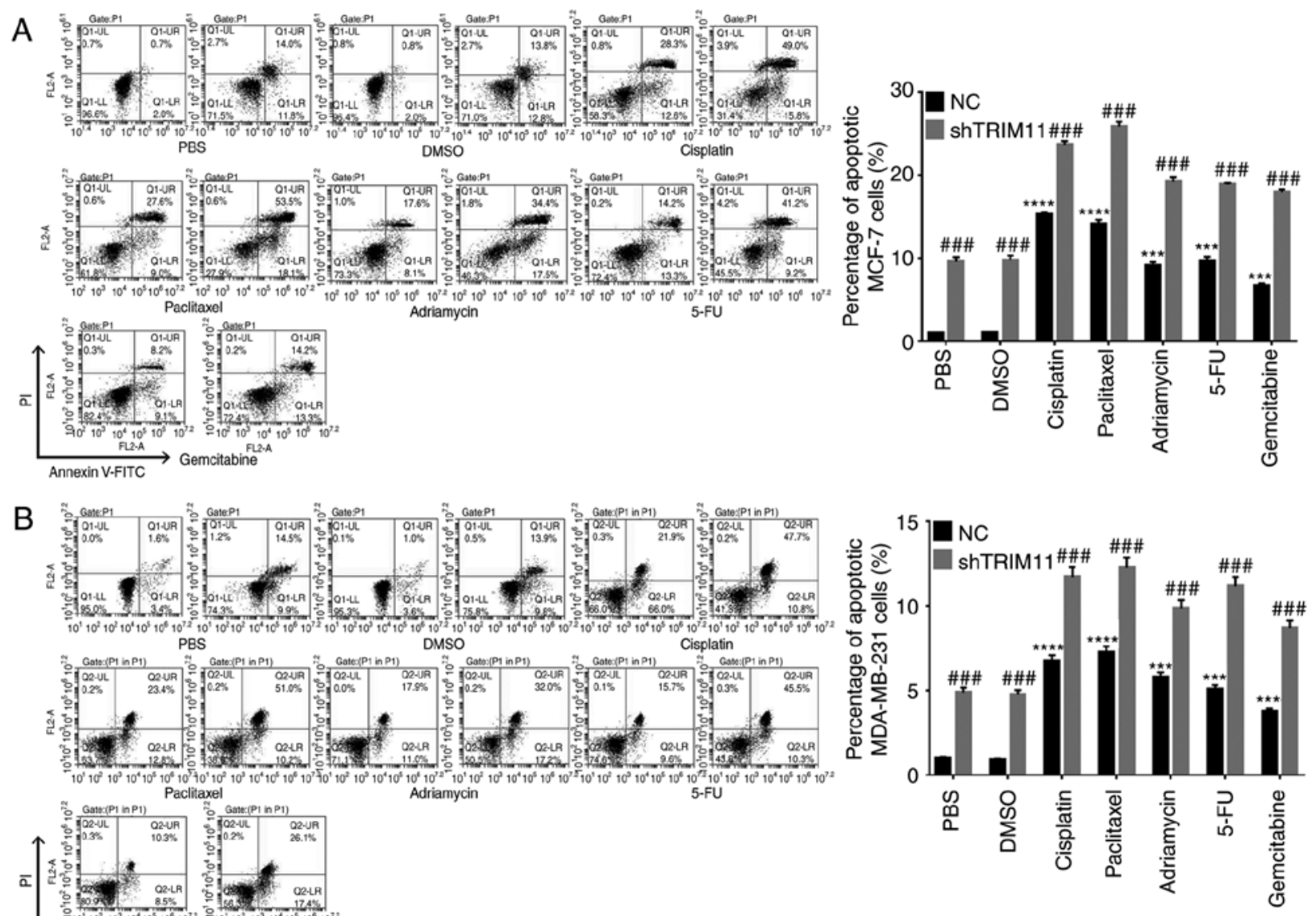

Figure 5. Downregulation of TRIM11 enhances the effects of chemotherapeutic drugs on the apoptosis of (A) MCF-7 and (B) MDA-MB-231 cells. Following infection with the shTRIM11 lentivirus, (A) MCF-7 and (B) MDA-MB-231 cells were individually treated with five chemotherapeutic drugs (5 $\mu \mathrm{g} / \mathrm{ml}$ ). After $24 \mathrm{~h}$, the cells were collected to determine their apoptosis rates by flow cytometric analysis. PBS and DMSO were used as vehicle controls. Data are presented as the mean \pm standard deviation. ${ }^{* * * *} \mathrm{P}<0.001,{ }^{* * * *} \mathrm{P}<0.0001$, compared with vehicle controls; ${ }^{\# \# *} \mathrm{P}<0.001$, compared with NC. TRIM11, tripartite motif-containing 11; NC, negative control; sh, short hairpin RNA; DMSO, dimethyl sulfoxide; 5-FU, fluorouracil.

associated with the alterations in TRIM11 expression, while ERK1/2 and JNK1/2 expression levels were unchanged. PTEN, p53 and Bax are pro-apoptotic proteins (25), and the ratio of $\mathrm{Bax} / \mathrm{Bcl}-2$ was reported to determine whether or not a cell was committed to apoptosis (26). All data revealed that TRIM11 may facilitate the proliferation of breast cancer cells through activating ERK1/2 and JNK1/2 signaling, which further regulates the expression of apoptosis-associated genes.

Downregulation of TRIM11 enhances the effects of chemotherapeutic drugs on the apoptosis of MCF-7 and MDA-MB-23I cells. Previous studies have demonstrated that the chemotherapeutic drugs cisplatin, paclitaxel, adriamycin, 5-FU and gemcitabine are widely used in the clinical treatment of breast cancer and other cancer types (5,9-13). Additionally, it was reported that $5 \mu \mathrm{M}$ cisplatin significantly inhibited the proliferation rate of breast cancer cells (27). To further investigate the effects of TRIM11, the aforementioned drugs were utilized and the cell apoptosis rates were measured after $24 \mathrm{~h}$ of treatment. As demonstrated in Fig. 5, in MCF-7 (Fig. 5A) and MDA-MB-231 (Fig. 5B) cells, cisplatin and paclitaxel had a more significant pro-apoptotic effect on cell apoptosis. Furthermore, following TRIM11 downregulation, the pro-apoptotic effects of these chemotherapeutic drugs were markedly stronger. Therefore, the results suggested that downregulation of TRIM11 can enhance the pro-apoptotic effects of chemotherapeutic drugs on breast cancer cells.

TRIM11 expression is increased in cisplatin- and paclitaxelresistant breast cancer tissues. A total of 4 pairs of cisplatin- and paclitaxel-sensitive and -resistant breast cancer tissues were collected to examine TRIM11 levels. As demonstrated in Fig. 6, a significant increase in the protein expression of TRIM11 was noted in the resistant tissues, whereas TRIM11 expression was much lower in the sensitive tissues. These data further suggested that TRIM11 downregulation enhanced the pro-apoptotic effects of chemotherapeutic drugs, which may be beneficial for the treatment of breast cancer.

\section{Discussion}

Although the breast is not an essential organ for human life, and breast cancer in situ is not fatal, the incidence of breast cancer has increased annually, causing serious damage to female physical and mental health. The pathophysiology of breast cancer has been studied for decades, but its cause requires further studies. The present study revealed that TRIM11 was important in the progression of breast cancer by 

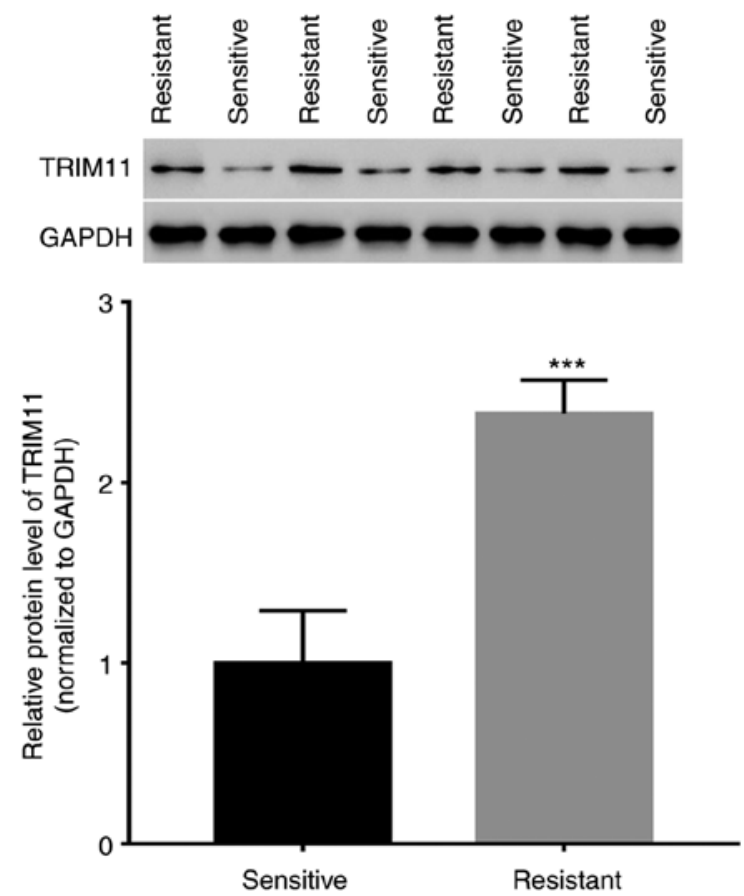

Figure 6. TRIM11 is increased in cisplatin- and paclitaxel-resistant breast cancer tissues. Cisplatin- and paclitaxel-sensitive and -resistant breast cancer tissues were obtained from patients and, following protein extraction, TRIM11 expression levels in the samples were detected by western blot analysis. All data are presented as the mean \pm standard deviation. ${ }^{* * *} \mathrm{P}<0.001$, compared with the drug-sensitive tissues. TRIM11, tripartite motif-containing 11.

regulating cell proliferation and apoptosis via activating the ERK1/2 and JNK1/2 signaling pathways.

Several members of the TRIM family have been revealed to function in breast cancer to date. For instance, knockdown of TRIM37, a breast cancer oncoprotein, decreases tumor growth (28). In addition, the proliferation of breast cancer cells was decreased due to the silencing of TRIM46 (29). In the present study, increased TRIM11 expression in breast cancer tissues may be associated with the progression of breast cancer; therefore, TRIM11 is likely to be a novel diagnostic indicator for breast cancer. In MCF-7 and MDA-MB-231 cells, downregulation of TRIM11 had an inhibitory effect on cell proliferation and a promoting effect on cell apoptosis, whereas TRIM11 overexpression resulted in opposite effects, which directly suggests an important role of TRIM11 in the proliferation and survival of breast cancer cells. Furthermore, PTEN, p53 and Bax expression levels were significantly increased with TRIM11 downregulation, while Bcl-2,p-ERK1/2 and p-JNK1/2 expression levels were decreased. PTEN, a lipid phosphatase, is a tumor suppressor that has been associated with several types of human cancer, and PTEN is frequently decreased in late-stage breast cancer (30). p53, also a tumor suppressor protein, may be a potential trans-regulator of Bcl-2 (31). A high expression level of Bcl-2 was detected in several solid tumor types, including breast cancer (32). Additionally, Bax is considered to be the main effector of apoptosis and functions in numerous types of human cancer (33). The binding site of p53 was identified in the promoter of the Bax gene, and Bax was strongly trans-activated by $553(34,35)$. In addition, signaling pathways, including ERK, JNK and p38 proved to be major determinants in various processes, including survival, apoptosis and proliferation (36,37). It has been reported that ERK1/2 (38) and JNK1/2 (39) may be associated with breast cancer, and that the expression levels of Bcl-2, Bax and p-ERK were affected by TRIM11 expression (19). Therefore, TRIM11 downregulation resulted in a beneficial effect on breast cancer possibly through activating ERK1/2 and JNK1/2 signaling, and regulating the expression of apoptosis-associated genes. These results are further supported by the observations that TRIM11 downregulation enhanced the pro-apoptotic effect of chemotherapeutic drugs, and that TRIM11 was increased in cisplatin- and paclitaxel-resistant breast cancer tissues.

In summary, the present study demonstrated that TRIM11 downregulation may improve the treatment of breast cancer by activating ERK1/2 and JNK1/2 signaling. TRIM11 may provide a novel direction for the study of the pathogenesis and treatment of breast cancer.

\section{Acknowledgements}

Not applicable.

\section{Funding}

No funding was received.

\section{Availability of data and materials}

All data generated or analyzed during this study are included in this published article.

\section{Authors' contributions}

XD and MiL conceived and designed the study. XD, FG and MeL performed the experiments. XD and MiL wrote the manuscript. All authors read and approved the final manuscript.

\section{Ethics approval and consent to participate}

All experiments conducted in the present study were approved by the Ethics Committee of Binzhou Medical University and written informed consent was obtained from all participants.

\section{Patient consent for publication}

Not applicable.

\section{Competing interests}

The authors declare that they have no competing interests.

\section{References}

1. Howlader N, Noone AM, Krapcho M, Garshell J, Miller D, Altekruse SF, Kosary CL, Yu M, Ruhl J, Tatalovich Z, et al: SEER cancer statistics review, 1975-2012, National Cancer Institute. 2013.

2. Demicheli R, Valagussa P and Bonadonna G: Does surgery modify growth kinetics of breast cancer micrometastases? Br J Cancer 85: 490-492, 2001.

3. Hansen E, Wolff N, Knuechel R, Ruschoff J, Hofstaedter F and Taeger K: Tumor cells in blood shed from the surgical field. Arch Surg 130: 387-393, 1995. 
4. Galluzzi L, Vitale I, Michels J, Brenner C, Szabadkai G, Harel-Bellan A, Castedo M and Kroemer G: Systems biology of cisplatin resistance: Past, present and future. Cell Death Dis 5: e1257, 2014.

5. Wang $\mathbf{J}$ and Wu GS: Role of autophagy in cisplatin resistance in ovarian cancer cells. J Biol Chem 289: 17163-17173, 2014.

6. Walsh V and Goodman J: From taxol to Taxol: The changing identities and ownership of an anti-cancer drug. Med Anthropol 21: 307-336, 2002

7. Liebmann JE, Cook JA, Lipschultz C, Teague D, Fisher J and Mitchell JB: Cytotoxic studies of paclitaxel (Taxol) in human tumour cell lines. Br J Cancer 68: 1104-1109, 1993.

8. Manfredi JJ and Horwitz SB: Taxol: An antimitotic agent with a new mechanism of action. Pharmacol Ther 25: 83-125, 1984.

9. Kampan NC, Madondo MT, Mcnally OM, Quinn M and Plebanski M: Paclitaxel and its evolving role in the management of ovarian cancer. Biomed Res Int 2015: 413076, 2015.

10. Pritchard JE, Dillon PM, Conaway MR, Silva CM and Parsons SJ: A mechanistic study of the effect of doxorubicin/adriamycin on the estrogen response in a breast cancer model. Oncology 83 305-320, 2012.

11. Bao L, Haque A, Jackson K, Hazari S, Moroz K, Jetly R and Dash S: Increased expression of P-glycoprotein is associated with doxorubicin chemoresistance in the metastatic 4T1 breast cancer model. Am J Pathol 178: 838-852, 2011.

12. Siena L, Pace E, Ferraro M, Di Sano C, Melis M, Profita M, Spatafora M and Gjomarkaj M: Gemcitabine sensitizes lung cancer cells to Fas/FasL system-mediated killing. Immunology 141: 242-255, 2014.

13. García MA, Carrasco E, Aguilera M, Alvarez P, Rivas C, Campos JM, Prados JC, Calleja MA, Esteban M, Marchal JA, et al: The chemotherapeutic drug 5-fluorouracil promotes PKR-mediated apoptosis in a p53-independent manner in colon and breast cancer cells. PLoS One 6: e23887, 2011

14. Abe O, Abe R, Enomoto K, Kikuchi K, Koyama H, Masuda H, Nomura Y, Sakai K, Sugimachi K, Tominaga T, et al: Effects of chemotherapy and hormonal therapy for early breast cancer on recurrence and 15-year survival: An overview of the randomised trials. Lancet 365: 1687-1717, 2005.

15. Tomar D and Singh R: TRIM family proteins: Emerging class of RING E3 ligases as regulator of NF- $\mathrm{KB}$ pathway. Biol Cell 107: 22-40, 2015 .

16. Hatakeyama S: TRIM proteins and cancer. Nat Rev Cancer 11: 792-804, 2011

17. Wang X, Shi W, Shi H, Lu S, Kang W, Chao S, He J, Jin W, Lv X, Zou H and Shu Y: TRIM11 overexpression promotes proliferation, migration and invasion of lung cancer cells. J Exp Clin Cancer Res 35: 100, 2016

18. Yin Y, Zhong J, Li SW, Li JZ, Zhou M, Chen Y, Sang Y and Liu L: TRIM11, a direct target of miR-24-3p, promotes cell proliferation and inhibits apoptosis in colon cancer. Oncotarget 7: 86755-86765, 2016.

19. Chen Y, Sun J and Ma J: Proliferation and invasion of ovarian cancer cells are suppressed by knockdown of TRIM11. Oncol Lett 14: 2125-2130, 2017

20. Chambon M, Orsetti B, Berthe ML, Bascoul-Mollevi C, Rodriguez C, Duong V, Gleizes M, Thénot S, Bibeau F, Theillet C and Cavaillès V: Prognostic significance of TRIM24/TIF-1 $\alpha$ gene expression in breast cancer. Am J Pathol 178: 1461-1469, 2011.

21. Tsai WW, Wang Z, Yiu TT, Akdemir KC, Xia W, Winter S, Tsai CY, Shi X, Schwarzer D, Plunkett W, et al: TRIM24 links a non-canonical histone signature to breast cancer. Nature 468 : 927-932, 2010
22. Ai L, Kim WJ, Alpay M, Ming T, Pardo CE, Hatakeyama S, May WS, Kladde MP, Heldermon CD, Siegel EM, et al: TRIM29 suppresses TWIST1 and invasive breast cancer behavior. Cancer Res 74: 4875-4887, 2014.

23. Livak KJ and Schmittgen TD: Analysis of relative gene expression data using real-time quantitative PCR and the $2^{-\Delta \Delta C \mathrm{~T}}$ method. Methods 25: 402-408, 2001.

24. Hong JY, Kang B, Kim A, Hwang S, Ahn J, Lee S, Kim J, Park JH and Cheon DS: Development of a highly sensitive real-time one step RT-PCR combined complementary locked primer technology and conjugated minor groove binder probe. Virol J 8: 330, 2011.

25. Gao $G$ and Dou QP: $G_{1}$ phase-dependent expression of Bcl-2 mRNA and protein correlates with chemoresistance of human cancer cells. Mol Pharmacol 58: 1001-1010, 2000.

26. Green DR and Reed JC: Mitochondria and apoptosis. Science 281: 1309-1312, 1998.

27. Bu Y, Lu C, Bian C, Wang J, Li J, Zhang B, Li Z, Brewer G and Zhao RC: Knockdown of Dicer in MCF-7 human breast carcinoma cells results in G1 arrest and increased sensitivity to cisplatin. Oncol Rep 21: 13-17, 2009.

28. Bhatnagar S, Gazin C, Chamberlain L, Ou J, Zhu X, Tushir JS, Virbasius CM, Lin L, Zhu LJ, Wajapeyee N and Green MR: TRIM37 is a new histone H2A ubiquitin ligase and breast cancer oncoprotein. Nature 516: 116-120, 2014.

29. Li Z, Li X, Wei D, Sun C, Guo D and Zhang L: Mmu-miR-1894-3p inhibits cell proliferation and migration of breast cancer cells by targeting Trim46. Int J Mol Sci 17: E609, 2016.

30. Li J, Yen C, Liaw D, Podsypanina K, Bose S, Wang SI, Puc J, Miliaresis C, Rodgers L, McCombie R, et al: PTEN, a putative protein tyrosine phosphatase gene mutated in human brain, breast, and prostate cancer. Science 275: 1943-1947, 1997.

31. Miyashita T, Harigai M, Hanada M and Reed JC: Identification of a p53-dependent negative response element in the $b c l-2$ gene. Cancer Res 54: 3131-3135, 1994.

32. Silvestrini R, Veneroni S, Daidone MG, Benini E, Boracchi $\mathrm{P}$, Mezzetti M, Di Fronzo G, Rilke F and Veronesi U: The Bcl-2 protein: A prognostic indicator strongly related to $\mathrm{p} 53$ protein in lymph node-negative breast cancer patients. J Natl Cancer Inst 86: 499-504, 1994.

33. Zhao G, Zhu Y, Eno CO, Liu Y, Deleeuw L, Burlison JA, Chaires JB, Trent JO and Li C: Activation of the proapoptotic Bcl-2 protein Bax by a small molecule induces tumor cell apoptosis. Mol Cell Biol 34: 1198-1207, 2014.

34. Miyashita T, Krajewski S, Krajewska M, Wang HG, Lin HK, Liebermann DA, Hoffman B and Reed JC: Tumor suppressor p53 is a regulator of bcl-2 and bax gene expression in vitro and in vivo. Oncogene 9: 1799-1805, 1994.

35. Miyashita T and Reed JC: Tumor suppressor p53 is a direct transcriptional activator of the human bax gene. Cell 80: 293-299, 1995.

36. Kohno $\mathrm{M}$ and Pouyssegur J: Targeting the ERK signaling pathway in cancer therapy. Ann Med 38: 200-211, 2006.

37. Lin A: Activation of the JNK signaling pathway: Breaking the brake on apoptosis. Bioessays 25: 17-24, 2003.

38. Nunes-Xavier CE, Elson A and Pulido R: Epidermal growth factor receptor (EGFR)-mediated positive feedback of protein-tyrosine phosphatase epsilon (PTPepsilon) on ERK1/2 and AKT protein pathways is required for survival of human breast cancer cells. J Biol Chem 287: 3433-3444, 2012.

39. Cellurale C, Girnius N, Jiang F, Cavanagh-Kyros J, Lu S, Garlick DS, Mercurio AM and Davis RJ: Role of JNK in mammary gland development and breast cancer. Cancer Res 72: 472-481, 2012. 